\title{
OPTIMAL CONTROL WITH ABSOLUTELY CONTINUOUS STRATEGIES FOR SPECTRALLY NEGATIVE LÉVY PROCESSES
}

\author{
ANDREAS E. KYPRIANOU,*** University of Bath \\ RONNIE LOEFFEN, ${ }^{* * *}$ Weierstrass Institute for Applied Analysis and Stochastics \\ JOSÉ-LUIS PÉREZ,***** University of Bath
}

\begin{abstract}
In the last few years there has been renewed interest in the classical control problem of de Finetti (1957) for the case where the underlying source of randomness is a spectrally negative Lévy process. In particular, a significant step forward was made by Loeffen (2008), who showed that a natural and very general condition on the underlying Lévy process which allows one to proceed with the analysis of the associated Hamilton-JacobiBellman equation is that its Lévy measure is absolutely continuous, having completely monotone density. In this paper we consider de Finetti's control problem, but with the restriction that control strategies are absolutely continuous with respect to the Lebesgue measure. This problem has been considered by Asmussen and Taksar (1997), JeanblancPicqué and Shiryaev (1995), and Boguslavskaya (2006) in the diffusive case, and Gerber and Shiu (2006) for the case of a Cramér-Lundberg process with exponentially distributed jumps. We show the robustness of the condition that the underlying Lévy measure has a completely monotone density and establish an explicit optimal strategy for this case that envelopes the aforementioned existing results. The explicit optimal strategy in question is the so-called refraction strategy.
\end{abstract}

Keywords: Scale function; ruin problem; de Finetti dividend problem; complete monotonicity

2010 Mathematics Subject Classification: Primary 60J99

Secondary 93E20; 60G51

\section{Introduction and main result}

Recently, there has been a growing body of literature which explores the interaction of classical models of ruin and fluctuation theory of Lévy processes; see, for example, [4], [8], [12], [17], [18], [20], [23], [24], and [25]-[28]. Of particular note in this respect is the application of the theory of scale functions for spectrally negative Lévy processes. This work adds to the aforementioned list by addressing a modification of de Finetti's classical dividend problem through the theory of scale functions. Before turning to our main results, let us first attend to the basic definitions of the mathematical objects that we are predominantly interested in.

\footnotetext{
Received 24 August 2010; revision received 23 August 2011.

* Postal address: Department of Mathematical Sciences, University of Bath, Claverton Down, Bath BA2 7AY, UK.

** Email address: a.kyprianou@bath.ac.uk

*** Current address: School of Mathematics, University of Manchester, Oxford Road, Manchester M13 9PL, UK.

R. L. gratefully acknowledges support from the AXA Research Fund.

**** Email address: jlapg20@bath.ac.uk

J.-L. P. acknowledges financial support from CONACyT, grant number 000000000129326.
} 
Recall that a spectrally negative Lévy process is a stochastic process issued from the origin which has càdlàg paths and stationary and independent increments such that there are no positive discontinuities. To avoid degenerate cases in the forthcoming discussion, we shall additionally exclude from this definition the case of monotone paths. This means that we are not interested in the case of a deterministic increasing linear drift or the negative of a subordinator.

Henceforth, we assume that $X=\left\{X_{t}: t \geq 0\right\}$ is a spectrally negative Lévy process under $\mathrm{P}$ with Lévy triplet given by $(\gamma, \sigma, v)$, where $\gamma \in \mathbb{R}, \sigma \geq 0$, and $v$ is a measure concentrated on $(0, \infty)$ satisfying

$$
\int_{(0, \infty)}\left(1 \wedge z^{2}\right) v(\mathrm{~d} z)<\infty .
$$

The Laplace exponent of $X$ is given by

$$
\psi(\lambda)=\log \mathrm{E}\left[\mathrm{e}^{\lambda X_{1}}\right]=\gamma \lambda+\frac{1}{2} \sigma^{2} \lambda^{2}-\int_{(0, \infty)}\left(1-\mathrm{e}^{-\lambda z}-\lambda z \mathbf{1}_{\{0<z \leq 1\}}\right) \nu(\mathrm{d} z),
$$

which is well defined for $\lambda \geq 0$. Here $\mathrm{E}$ denotes the expectation with respect to $\mathrm{P}$. The reader will note that, for convenience, we have arranged the representation of the Laplace exponent in such a way that the support of the Lévy measure is positive even though the process experiences only negative jumps. As a strong Markov process, we shall endow $X$ with probabilities $\left\{\mathrm{P}_{x}: x \in \mathbb{R}\right\}$ such that, under $\mathrm{P}_{x}$, we have $X_{0}=x$ with probability 1 . Note that $\mathrm{P}_{0}=\mathrm{P}$.

It is well known that $X$ has paths of bounded variation if and only if $\sigma=0$ and $\int_{0}^{1} z \nu(\mathrm{d} z)<\infty$. In this case $X$ can be written as

$$
X_{t}=c t-S_{t}, \quad t \geq 0,
$$

where $c=\gamma+\int_{0}^{1} z v(\mathrm{~d} z)$ and $\left\{S_{t}: t \geq 0\right\}$ is a driftless subordinator. Note that necessarily $c>0$ and $v \not \equiv 0$, since we have ruled out the case that $X$ has monotone paths. Moreover, when $v(0, \infty)<\infty$, then $X$ is known in the actuarial mathematics literature as the classical Cramér-Lundberg risk process. This process is often used to model the surplus wealth of an insurance company.

The classical theory of ruin concerns itself with the path of the stochastic risk process until the moment that it first passes below the level 0; the event corresponding to ruin. An offshoot of the classical ruin problem was introduced by de Finetti [11]. His intention was to make the study of ruin more realistic by introducing the possibility that dividends are paid out to shareholders up to the moment of ruin. Furthermore, the payment of dividends should be made in such a way as to optimize the expected net present value of the total dividends paid to the shareholders from time 0 until ruin. Mathematically speaking, de Finetti's dividend problem amounts to solving a control problem which we state in the next paragraph. Although de Finetti's dividend problem has its origin in insurance mathematics, there are several papers [6], [7], [30] that have considered this problem in the context of corporate finance.

Let $\pi=\left\{L_{t}^{\pi}: t \geq 0\right\}$ be a dividend strategy, meaning that it is a left-continuous, nonnegative, nondecreasing process adapted to the (completed and right-continuous) filtration $\mathbb{F}:=\left\{\mathscr{F}_{t}: t \geq\right.$ 0 \} of $X$. The quantity $L_{t}^{\pi}$ thus represents the cumulative dividends paid out up to time $t$ by the insurance company whose risk process is modelled by $X$. An additional constraint on $\pi$ is that $L_{t+}^{\pi}-L_{t}^{\pi} \leq \max \left\{U_{t}^{\pi}, 0\right\}$ for $t \geq 0$ (i.e. lump sum dividend payments are always smaller than the available reserves). The $\pi$-controlled Lévy process is thus $U^{\pi}=\left\{U_{t}^{\pi}: t \geq 0\right\}$, where $U_{t}^{\pi}=X_{t}-L_{t}^{\pi}$. Write $\sigma^{\pi}=\inf \left\{t>0: U_{t}^{\pi}<0\right\}$ for the time at which ruin occurs when the 
dividend payments are taken into account. Suppose that $\Pi$ denotes some family of admissible strategies, which we shall elaborate on later. Then the expected net present value of the dividend policy $\pi \in \Pi$ with discounting at rate $q>0$ and initial capital $x \geq 0$ is given by

$$
v_{\pi}(x)=\mathrm{E}_{x}\left[\int_{\left[0, \sigma^{\pi}\right]} \mathrm{e}^{-q t} \mathrm{~d} L_{t}^{\pi}\right],
$$

where $\mathrm{E}_{x}$ denotes the expectation with respect to $\mathrm{P}_{x}$ and $q>0$ is a fixed rate. De Finetti's dividend problem consists of characterising the optimal value function,

$$
v_{*}(x):=\sup _{\pi \in \Pi} v_{\pi}(x),
$$

and, further, if it exists, establishing a strategy $\pi^{*}$ such that $v_{*}(x)=v_{\pi^{*}}(x)$.

In the case that $\Pi$ consists of all strategies as described at the beginning of the previous paragraph, de Finetti's dividend problem belongs to the class of singular stochastic control problems; the term 'singular' refers to the property that the controls are allowed to be singular (with respect to the Lebesgue measure) in time. For this case, there are now extensive results in the literature, most of which have appeared in the last few years. Initially, this problem was considered by Gerber [14], who proved that, for the Cramér-Lundberg model with exponentially distributed jumps, the optimal value function is the result of a reflection strategy. That is to say, a strategy of the form $L_{t}^{a}=\left(a \vee \bar{X}_{t}\right)-a$ for some, optimally chosen, barrier $a \geq 0$, where $\bar{X}_{t}:=\sup _{s \leq t} X_{s}$. In that case the controlled process $U_{t}^{a}=X_{t}-L_{t}^{a}$ is a spectrally negative Lévy process reflected at the barrier $a$. However, a sequence of innovative works [4], [5], [24], [25], [26], [28] have pushed this conclusion much further into the considerably more general setting where $X$ is a spectrally negative Lévy process. Of particular note amongst these references is the paper of Loeffen [25] in which the optimality of the reflection strategy is shown to depend in a very subtle way on the shape of the so-called scale functions associated to the underlying Lévy process. Indeed, Loeffen's new perspective on de Finetti's control problem leads to very easily verifiable sufficient conditions for the reflection strategy to be optimal. Loeffen showed that it suffices for the Lévy measure $v$ to be absolutely continuous with a completely monotone density. Though this assumption seems quite restrictive at first sight, there are actually plenty of examples of general spectrally negative Lévy processes that are used in risk theory and satisfy this assumption; see [25, pp. 1677-1678]. Through largely technical adaptations of Loeffen's method, this sufficient condition was relaxed in [24] and [28]. It is important to note that in general a barrier strategy is not always an optimal strategy; an explicit counterexample was provided in [5].

In this paper we are interested in addressing an adaptation of de Finetti's dividend problem by considering a smaller class of admissible strategies. Specifically, we are interested in the case where, in addition to the assumption that strategies are nondecreasing and $\mathcal{F}$-adapted, $\Pi$ only admits absolutely continuous strategies $\pi=\left\{L_{t}^{\pi}: t \geq 0\right\}$ such that

$$
L_{t}^{\pi}=\int_{0}^{t} \ell^{\pi}(s) \mathrm{d} s,
$$

and, for $t \geq 0, \ell^{\pi}(t)$ satisfies

$$
0 \leq \ell^{\pi}(t) \leq \delta
$$

where $\delta>0$ is a ceiling rate. Moreover, we make the following assumption.

(H) $\delta<\gamma+\int_{0}^{1} z v(\mathrm{~d} z)$ if $X$ has paths of bounded variation. 
Note that, for a reflection strategy, when $X$ has paths of unbounded variation, the corresponding dividend process is supported by increase times that are singular with respect to the Lebesgue measure, and when $X$ has paths of bounded variation, the dividend process is supported by increase times which are absolutely continuous with respect to the Lebesgue measure with rate $c$ (cf. Section 6.1 of [21]). Recalling decomposition (1) and assumption (H), we see that the reflection strategy is therefore not included in the smaller class of admissible controls and we are left with a truly different control problem. A particular motivation for studying this kind of modification is that if a reflection strategy is applied then the company will be ruined in finite time with probability 1 , which is seen as an undesirable consequence; cf. [15]. By restricting the set of admissible strategies in the way described above and provided that $\mathrm{E}\left[X_{1}\right]>\delta$, we make sure that there is a strictly positive probability that ruin will never occur no matter which admissible dividend strategy is applied.

The reader familiar with optimal control problems of this kind will recognize that the optimal strategy should be of bang-bang type, i.e. depending on the value of the controlled process, dividends should either be paid out at the maximum rate $\delta$ or at the minimum rate 0 . A particularly simple bang-bang strategy is the one that we refer to here as a refraction strategy, which, in words, is the strategy where dividends are paid out at the maximum rate when the controlled process is above a certain level $b \geq 0$ and at the minimum rate when below $b$. Mathematically, a refraction strategy at $b$ is the strategy which corresponds to the controlled process taking the form of the unique strong solution to the following stochastic differential equation:

$$
\mathrm{d} U_{t}^{b}=\mathrm{d} X_{t}-\delta \mathbf{1}_{\left\{U_{t}^{b}>b\right\}} \mathrm{d} t, \quad t \geq 0 .
$$

In the case of absolutely continuous control strategies for $X$, it has been shown by Asmussen and Taksar [2], Jeanblanc-Picqué and Shiryaev [19], and Boguslavskaya [9] in the diffusive case, and by Gerber and Shiu [16] for the case of a Cramér-Lundberg process with exponentially distributed jumps that a refraction strategy, where $b \geq 0$ is optimally chosen, is optimal. This particular control problem is also discussed in the review papers of Avanzi [3] and Albrecher and Thonhauser [1], and in the book of Schmidli [31].

In the spirit of earlier work for the more general class of admissible strategies, the point of view we shall take here is to deal with a general spectrally negative Lévy process and give sufficient conditions under which a refraction strategy of the form (5) is optimal. Note that when $X$ is a general spectrally negative Lévy process, the strong existence and uniqueness of solutions to (5) under $(\mathrm{H})$, so-called refracted Lévy processes, were established in [22]. Our main result is the following.

Theorem 1. Suppose that the Lévy measure has a completely monotone density. Let $\Pi$ be the class of admissible dividend strategies satisfying (3), (4), and (H). Then an optimal strategy, i.e. a strategy which attains the supremum in (2), is formed by a refraction strategy.

Given the special cases for which Theorem 1 is already known, recent developments on the singular version of de Finetti's problem, and recent developments concerning refracted Lévy processes, the statement of Theorem 1 (and the nature of its proof) should not come as a great surprise. However, a particular step of the proof, namely Lemma 7 below, turned out to be quite a difficult puzzle to solve. This is mainly due to the fact that the expression, written in terms of scale functions, for the value function of a refraction strategy is substantially more complicated than that of a reflection strategy; compare Equation (10.25) of [22] with Proposition 1 of [4]. The above theorem offers the same sufficient condition on the Lévy measure as Loeffen [25] for the larger, general class of admissible strategies. Although, as alluded to above, weaker 
assumptions have been established in that case, the technical details of our method appear not to allow us to follow suit. To illustrate the difference between the two cases, we give in Example 1 a specific example of $X$ and $q$ for which no refraction strategy can be optimal in the restricted case for a certain choice of the ceiling rate $\delta$, whereas a reflection strategy is optimal within the general class of admissible dividend strategies. We also remark that in fact our method allows us to give a more quantitative result than Theorem 1 in the sense that we are able to characterise the threshold $b^{*}$ associated with the optimal refraction strategy. As some more notation is needed to do this, it is given at the end of the paper in Corollary 1.

We close this section with a brief summary of the remainder of the paper. In the next section we show the role played by scale functions in giving a workable identity for the expected net present value of the paid out dividends until ruin in the case where a refraction strategy is applied. We also use this identity to describe an appropriate candidate for the threshold associated with the optimal refraction strategy. Then in the final section we put together a series of technical lemmas which allow us to verify the optimality of the identified threshold strategy. The assumption that $v$ has a completely monotone density will repeatedly play a very significant role in the aforementioned lemmas.

\section{Scale functions and refraction strategies}

As alluded to above, a key element of the forthcoming analysis relies on the theory of socalled scale functions. We therefore devote some time in this section reminding the reader of some fundamental properties of scale functions as well as their relevance to refraction strategies.

For each $q \geq 0$, the so-called $q$-scale function of $X, W^{(q)}: \mathbb{R} \rightarrow[0, \infty)$, is the unique function such that $W^{(q)}(x)=0$ for $x<0$, and on $[0, \infty)$ is a strictly increasing and continuous function whose Laplace transform is given by

$$
\int_{0}^{\infty} \mathrm{e}^{-\theta x} W^{(q)}(x) \mathrm{d} x=\frac{1}{\psi(\theta)-q}, \quad \theta>\Phi(q) .
$$

Here

$$
\Phi(q)=\sup \{\lambda \geq 0: \psi(\lambda)=q\},
$$

which is well defined and finite for all $q \geq 0$ as a consequence of the well-known fact that $\psi$ is a strictly convex function satisfying $\psi(0)=0$ and $\psi(\infty)=\infty$. Note that there is an abuse of notation here as the parameter $q$ of the scale functions has also been used to denote the discount rate. However, since we will only need the $q$-scale function (and $\Phi(q)$ ) with the corresponding parameter $q$ being equal to the discount rate, this should not cause too much confusion.

Shape and smoothness properties of the scale functions $W^{(q)}$ will be of particular interest in the forthcoming analysis. In the discussion below we shall consider the behaviour of $W^{(q)}$ at the origin and infinity as well as describing qualitative features of its shape on $(0, \infty)$. We start with some standard facts concerning the behaviour of the scale function in the neighbourhood of the origin. Recall that we have defined the constant

$$
c=\gamma+\int_{0}^{1} z v(\mathrm{~d} z)
$$

in the case that $X$ has bounded variation paths.

The following result is well known and can easily be deduced from (6). See, for example, Chapter 8 of [21]. 
Lemma 1. As $x \downarrow 0$, the value of the scale function $W^{(q)}(x)$ and its right derivative are determined for every $q \geq 0$ as follows:

$$
\begin{aligned}
W^{(q)}(0+) & = \begin{cases}\frac{1}{c} & \text { when } \sigma=0 \text { and } \int_{0}^{1} z v(\mathrm{~d} z)<\infty, \\
0 & \text { otherwise, }\end{cases} \\
W^{(q)^{\prime}}(0+) & = \begin{cases}\frac{2}{\sigma^{2}} & \text { when } \sigma>0, \\
\frac{v(0, \infty)+q}{c^{2}} & \text { when } \sigma=0 \text { and } v(0, \infty)<\infty, \\
\infty & \text { otherwise. }\end{cases}
\end{aligned}
$$

In general, it is known that one may always write, for $q \geq 0$,

$$
W^{(q)}(x)=\mathrm{e}^{\Phi(q) x} W_{\Phi(q)}(x)
$$

where $W_{\Phi(q)}$ plays the role of a 0 -scale function of an auxiliary spectrally negative Lévy process with Laplace exponent given by $\psi_{\Phi(q)}(\lambda)=\psi(\lambda+\Phi(q))-q$. Note that the fact that $\psi_{\Phi(q)}$ is the Laplace exponent follows by an exponential tilting argument; see, for example, Chapter 8 of [21]. In the same reference we also see that $\lim _{x \uparrow \infty} W_{\Phi(q)}(x)<1 / \psi^{\prime}(\Phi(q))<\infty$, which suggests that, when $q>0$, the function $W^{(q)}(x)$ behaves like the exponential function $\mathrm{e}^{\Phi(q) x}$ for large $x$. It is therefore natural to ask whether $W^{(q)}(x)$ is convex for large values of $x$. This very question was addressed by Loeffen [25], [26]. In these papers it was found that, due to quite a deep connection between scale functions and potential measures of subordinators, a natural assumption which allows one to address the issue of convexity, and, in fact, say a lot more (cf. Lemma 2 below), is that the Lévy measure $v$ is absolutely continuous with completely monotone density. In the next lemma we collect a number of consequences of this assumption, lifted from the aforementioned two papers. We first need some more notation. Recalling that $W^{(q)}$ is continuously differentiable on $(0, \infty)$ as soon as $v$ has no atoms (see, for example, the discussion in [10]), a key quantity in the lemma is the constant

$$
a^{*}=\sup \left\{a \geq 0: W^{(q)^{\prime}}(a) \leq W^{(q)^{\prime}}(x) \text { for all } x \geq 0\right\},
$$

where $W^{(q) \prime}(0)$ stands for $W^{(q) \prime}(0+)$. Furthermore, $a^{*}<\infty$ since, by (7), we have $\lim _{x \uparrow \infty} W^{(q) \prime}(x)=\infty$. We record here the following result taken from [26, Theorem 2 and Corollary 1]; note that $\Phi^{\prime}(q)=1 / \psi^{\prime}(\Phi(q))$.

Lemma 2. Suppose that the Lévy measure has a completely monotone density and $q>0$. Then the $q$-scale function can be written as

$$
W^{(q)}(x)=\Phi^{\prime}(q) \mathrm{e}^{\Phi(q) x}-f(x), \quad x>0,
$$

where $f$ is a nonnegative, completely monotone function. Moreover, $W^{(q) \prime}$ is strictly log-convex (and, hence, convex) on $(0, \infty)$. Since $W^{(q)^{\prime}}(\infty)=\infty, a^{*}$ is thus the unique point at which $W^{(q) \prime}$ attains its minimum so that $W^{(q) \prime}$ is strictly decreasing on $\left(0, a^{*}\right)$ and strictly increasing on $\left(a^{*}, \infty\right)$.

Let us now progress to a description of the role played by scale functions in connection with the value of a refraction strategy. In addition to the scale function $W^{(q)}$ associated to the spectrally negative Lévy process $X$, we shall also define, for each $q \geq 0$, the scale functions $\mathbb{W}^{(q)}$ which are associated to the linearly perturbed spectrally negative Lévy process $Y=\left\{Y_{t}: t \geq 0\right\}$, where $Y_{t}=X_{t}-\delta t$ for $t \geq 0$; recall that $\delta$ stands for the ceiling rate. Note that, because of 
assumption $(\mathrm{H})$, the aforementioned process does not have monotone paths. Furthermore, we denote by $\varphi(q)$ the right inverse of the Laplace exponent of $Y$, i.e.

$$
\varphi(q)=\inf \{\lambda \geq 0: \psi(\lambda)-\delta \lambda=q\} .
$$

The value function of the refraction strategy at level $b$, henceforth denoted by $v_{b}$, can now be written explicitly in terms of $W^{(q)}, \mathbb{W}^{(q)}$, and $\varphi(q)$ with the parameter $q>0$ being the discount rate. Indeed, it was shown in Equation (10.25) of [22] that

$$
v_{b}(x)=-\delta \int_{0}^{x-b} \mathbb{W}^{(q)}(y) \mathrm{d} y+\frac{W^{(q)}(x)+\delta \int_{b}^{x} \mathbb{W}^{(q)}(x-y) W^{(q) \prime}(y) \mathrm{d} y}{h(b)}, \quad x \geq 0,
$$

where $h(b)$ is given by

$$
h(b)=\varphi(q) \mathrm{e}^{\varphi(q) b} \int_{b}^{\infty} \mathrm{e}^{-\varphi(q) y} W^{(q) \prime}(y) \mathrm{d} y=\varphi(q) \int_{0}^{\infty} \mathrm{e}^{-\varphi(q) u} W^{(q) \prime}(u+b) \mathrm{d} u .
$$

Note that

$$
v_{b}(x)=\frac{W^{(q)}(x)}{h(b)} \quad \text { for } x \leq b .
$$

We also need to have a candidate optimal threshold, say $b^{*}$, in combination with the expression for $v_{b}$ if we are to check for optimality. To this end, define $b^{*}$ as the largest argument at which $h$ attains its minimum. That is,

$$
b^{*}=\sup \{b \geq 0: h(b) \leq h(x) \text { for all } x \geq 0\} .
$$

Under the same conditions as Theorem 1, we are able to say some more about $b^{*}$.

Lemma 3. Suppose that $v$ has a completely monotone density. Then $b^{*} \in\left[0, a^{*}\right)$ and it is the unique point at which $h$ attains its minimum. Moreover, $b^{*}>0$ if and only if one of the following three cases hold:

(i) $\sigma>0$ and $\varphi(q)<2 \delta / \sigma^{2}$,

(ii) $\sigma=0, v(0, \infty)<\infty$, and $\varphi(q)<\delta(v(0, \infty)+q) / c(c-\delta)$, or

(iii) $\sigma=0$ and $v(0, \infty)=\infty$.

Proof. We begin by showing that $b^{*}<\infty$. Note that

$$
\begin{aligned}
h(b) & =(\varphi(q))^{2} \int_{0}^{\infty} \mathrm{e}^{-\varphi(q) y}\left(W^{(q)}(y+b)-W^{(q)}(b)\right) \mathrm{d} y \\
& =(\varphi(q))^{2} \mathrm{e}^{\Phi(q) b} \int_{0}^{\infty} \mathrm{e}^{-\varphi(q) y}\left(\mathrm{e}^{\Phi(q) y} W_{\Phi(q)}(y+b)-W_{\Phi(q)}(b)\right) \mathrm{d} y \\
& \geq(\varphi(q))^{2} \mathrm{e}^{\Phi(q) b} W_{\Phi(q)}(b) \int_{0}^{\infty} \mathrm{e}^{-\varphi(q) y}\left(\mathrm{e}^{\Phi(q) y}-1\right) \mathrm{d} y \\
& =W^{(q)}(b) \frac{\varphi(q) \Phi(q)}{\varphi(q)-\Phi(q)},
\end{aligned}
$$

where we have used a change of variables and an integration by parts for the first equality, and (7) for the second and third. Since $W^{(q)}(\infty)=\infty$ and $\varphi(q)>\Phi(q)$, it follows that 
$\lim _{b \rightarrow \infty} h(b)=\infty$. The latter implies that $b^{*}<\infty$ as $b^{*}$ is defined as the supremum of all the global minimizers of $h$.

From (9), we see that $h$ is continuously differentiable and that

$$
h^{\prime}(b)=\varphi(q)\left(h(b)-W^{(q) \prime}(b)\right) .
$$

It follows immediately that $h^{\prime}(b)>0$ if and only if $h(b)>W^{(q)^{\prime}}(b)$ and $h^{\prime}(b)<0$ if and only if $h(b)<W^{(q)^{\prime}}(b)$. Thanks to Lemma 2 , we know that $W^{(q)^{\prime}}$ is a strictly convex function satisfying $W^{(q)^{\prime}}(\infty)=\infty$, and, since $h(\infty)=\infty$, it follows that there is a unique $b^{*} \in[0, \infty)$ for which the minimum of $h$ is attained and $h(b)<W^{(q) \prime}(b)$ for $b<b^{*}$ and $h(b)>W^{(q)^{\prime}}(b)$ for $b>b^{*}$. Moreover, when $b^{*}>0$, we have $h\left(b^{*}\right)=W^{(q)^{\prime}}\left(b^{*}\right)$.

Let us now show that $b^{*}<a^{*}$. Suppose for contradiction that $b^{*}>a^{*}$. In that case, since $W^{(q) \prime \prime}(b)>0$ for all $b \geq b^{*}$ and $h^{\prime}\left(b^{*}\right)=0$, it follows that there exists a sufficiently small $\varepsilon>0$ such that $W^{(q)^{\prime}}(b)>h(b)$ for all $b \in\left(b^{*}, b^{*}+\varepsilon\right)$. However, this last statement contradicts the earlier conclusion that $W^{(q) \prime}(b)<h(b)$ for all $b>b^{*}$. Now suppose, also for contradiction, that $b^{*}=a^{*}$. Considering the second equality in (9), since $W^{(q)^{\prime}}\left(u+a^{*}\right)>W^{(q)^{\prime}}\left(a^{*}\right)$ for all $u>0$, it is straightforward to show that $h\left(a^{*}\right)>W^{(q)^{\prime}}\left(a^{*}\right)$, which again contradicts our earlier conclusion that $h\left(b^{*}\right)=W^{(q)}\left(b^{*}\right)$.

Finally, given that $b^{*}$ characterises the single crossing point of the function $h$ over the function $W^{(q) \prime}$, we have $b^{*}>0$ if and only if $h(0)<W^{(q) \prime}(0+)$. Note from (9) that

$$
h(0)=\varphi(q)\left(\frac{\varphi(q)}{\psi(\varphi(q))-q}-W^{(q)}(0)\right)=\varphi(q)\left(\frac{1}{\delta}-W^{(q)}(0)\right),
$$

where we have used the facts that, for $q>0$, by integration by parts in (6),

$$
\int_{[0, \infty)} \mathrm{e}^{-\theta x} W^{(q)}(\mathrm{d} x)=\frac{\theta}{\psi(\theta)-q}, \quad \theta>\Phi(q),
$$

and that $\varphi(q)>\Phi(q)$. The three cases that are equivalent to $b^{*}>0$ now follow directly from the right-hand side of (12) compared with the expression given for $W^{(q) \prime}(0+)$ in Lemma 1.

\section{Verification}

For the remainder of the paper, we will focus on verifying the optimality of the refraction strategy at threshold level $b^{*}$ under the condition that $v$ has a completely monotone density.

Given the spectrally negative Lévy process $X$, we call a function $f$ (defined on at least the positive half-line) sufficiently smooth if $f$ is continuously differentiable on $(0, \infty)$ when $X$ has paths of bounded variation and is twice continuously differentiable on $(0, \infty)$ when $X$ has paths of unbounded variation. We let $\Gamma$ be the operator acting on sufficiently smooth functions $f$, defined by

$$
\Gamma f(x)=\gamma f^{\prime}(x)+\frac{\sigma^{2}}{2} f^{\prime \prime}(x)+\int_{(0, \infty)}\left[f(x-z)-f(x)+f^{\prime}(x) z \mathbf{1}_{\{0<z \leq 1\}}\right] v(\mathrm{~d} z) .
$$

The following lemma constitutes standard technology as far as optimal control is concerned. For this reason, its proof, which requires only a technical modification of Lemma 1 of [26], is deferred to Appendix A. 
Lemma 4. Suppose that $\hat{\pi}$ is an admissible dividend strategy such that $v_{\hat{\pi}}$ is sufficiently smooth on $(0, \infty)$, right continuous at 0 , and, for all $x>0$,

$$
\sup _{0 \leq r \leq \delta} \Gamma v_{\hat{\pi}}(x)-q v_{\hat{\pi}}(x)-r v_{\hat{\pi}}^{\prime}(x)+r \leq 0 .
$$

Then $v_{\hat{\pi}}(x)=v_{*}(x)$ for all $x \geq 0$ and, hence, $\hat{\pi}$ is an optimal strategy.

As we wish to work with this lemma for the case that $v_{\hat{\pi}}=v_{b *}$, we show next that $v_{b^{*}}$ is sufficiently smooth.

Lemma 5. Under the assumption of Theorem 1, the value function $v_{b^{*}}$ is sufficiently smooth.

Proof. Recall from Lemma 2 that when $v$ has a completely monotone density, it follows that both $W^{(q)}$ and $\mathbb{W}^{(q)}$ are infinitely differentiable.

Now suppose that $b^{*}=0$. Then from (8) it follows that

$$
v_{0}(x)=-\delta\left(\int_{0}^{x} \mathbb{W}^{(q)}(y) \mathrm{d} y-\frac{1}{\varphi(q)} \mathbb{W}^{(q)}(x)\right), \quad x \geq 0,
$$

which is clearly sufficiently smooth.

Next suppose that $b^{*}>0$. By differentiating (8) we obtain

$$
v_{b^{*}}^{\prime}(x)=-\delta \mathbb{W}^{(q)}\left(x-b^{*}\right)+\frac{\left(1+\delta \mathbb{W}^{(q)}(0)\right) W^{(q) \prime}(x)+\delta \int_{b^{*}}^{x} \mathbb{W}^{(q) \prime}(x-y) W^{(q) \prime}(y) \mathrm{d} y}{W^{(q) \prime}\left(b^{*}\right)} .
$$

Using an integration by parts in (16) leads to

$$
v_{b^{*}}^{\prime}(x)=\frac{W^{(q) \prime}(x)+\delta \int_{b^{*}}^{x} \mathbb{W}^{(q)}(x-y) W^{(q) \prime \prime}(y) \mathrm{d} y}{W^{(q)^{\prime}}\left(b^{*}\right)},
$$

which is continuous in $x$. Differentiating (17) leads to

$$
v_{b^{*}}^{\prime \prime}(x)=\frac{W^{(q) \prime \prime}(x)+\delta \mathbb{W}^{(q)}(0) W^{(q) \prime \prime}(x)+\delta \int_{b^{*}}^{x} \mathbb{W}^{(q) \prime}(x-y) W^{(q) \prime \prime}(y) \mathrm{d} y}{W^{(q)^{\prime}}\left(b^{*}\right)} .
$$

The expression on the right-hand side is clearly continuous in $x$ when $X$ has paths of unbounded variation as $\mathbb{W}^{(q)}(0)=0$.

Inspired by the cases that $X$ is diffusive or a Cramér-Lundberg process with exponentially distributed jumps, for which a solution to the control problem at hand is known, we move next to the following two lemmas which convert the Hamilton-Jacobi-Bellman inequality in Lemma 4 into a more user friendly sufficient condition.

Lemma 6. Under the assumption of Theorem 1, the value function $v_{b^{*}}$ satisfies (14) if and only if

$$
v_{b^{*}}^{\prime}(x) \begin{cases}\geq 1 & \text { if } 0<x \leq b^{*} \\ \leq 1 & \text { if } x>b^{*}\end{cases}
$$

Proof. We first establish the following two equalities:

$$
\begin{gathered}
(\Gamma-q) v_{b^{*}}(x)=0 \text { for } 0<x \leq b^{*}, \\
(\Gamma-q) v_{b^{*}}(x)-\delta v_{b^{*}}^{\prime}(x)+\delta=0 \text { for } x>b^{*} .
\end{gathered}
$$


Recalling (10) and the fact that $v_{b^{*}}$ is sufficiently smooth, the first part of (20) is proved in Lemma 4 of [4] (see also [8]). In a similar way, the second part follows after we show that $M=\left\{M_{t}, t \geq 0\right\}$ given by

$$
M_{t}=\mathrm{e}^{-q\left(t \wedge \tau_{b^{*}}^{-}\right)}\left(v_{b^{*}}\left(Y_{t \wedge \tau_{b^{*}}^{-}}\right)-\frac{\delta}{q}\right), \quad t \geq 0,
$$

is a $\mathbb{P}_{x}$-martingale for $x>b^{*}$; here $\tau_{b^{*}}^{-}$stands for $\tau_{b^{*}}^{-}=\inf \left\{t>0: Y_{t}<b^{*}\right\}$ and $\mathbb{P}_{x}$ is the law of $Y$ when $Y_{0}=x$. Indeed, the martingale property follows by the two proceeding computations and the tower property of conditional expectation (cf. [33, Section 9.7]). First we have, for $x>b^{*}$ by the strong Markov property,

$$
\begin{aligned}
\mathbb{E}_{x}\left[\mathrm{e}^{-q \tau_{b^{*}}^{-}}\left(v_{b^{*}}\left(Y_{\tau_{b^{*}}^{-}}\right)-\frac{\delta}{q}\right) \mid \mathcal{F}_{t}\right]= & \mathbb{E}_{x}\left[\mathbf{1}_{\left\{t<\tau_{\left.b^{*}\right\}}^{-}\right.} \mathrm{e}^{-q \tau_{b^{*}}^{-}}\left(v_{b^{*}}\left(Y_{\tau_{b^{*}}^{-}}\right)-\frac{\delta}{q}\right) \mid \mathcal{F}_{t}\right] \\
& +\mathbb{E}_{x}\left[\mathbf{1}_{\left\{t \geq \tau_{b^{*}}^{-}\right\}} \mathrm{e}^{-q \tau_{b^{*}}}\left(v_{b^{*}}\left(Y_{\tau_{b^{*}}}\right)-\frac{\delta}{q}\right) \mid \mathcal{F}_{t}\right] \\
= & \mathbf{1}_{\left\{t<\tau_{\left.b^{*}\right\}}^{-}\right.} \mathrm{e}^{-q t} \mathbb{E}_{Y_{t}}\left[\mathrm{e}^{-q \tau_{b^{*}}^{-}}\left(v_{b^{*}}\left(Y_{\tau_{b^{*}}^{-}}\right)-\frac{\delta}{q}\right)\right] \\
& +\mathbf{1}_{\left\{t \geq \tau_{b^{*}}^{-}\right\}} M_{t} .
\end{aligned}
$$

Here $\mathbb{E}_{x}$ denotes the expectation with respect to $\mathbb{P}_{x}$. Recall that $U^{b^{*}}$ is the refracted Lévy process given by (5) with threshold $b^{*}$. Let

$$
\sigma^{b^{*}}=\inf \left\{t>0: U_{t}^{b^{*}}<0\right\} \quad \text { and } \quad \kappa_{b^{*}}^{-}=\inf \left\{t>0: U_{t}^{b^{*}}<b^{*}\right\} .
$$

Then

$$
\begin{aligned}
\mathbf{1}_{\left\{t<\tau_{\left.b^{*}\right\}}^{-}\right.} M_{t} & =\mathbf{1}_{\left\{t<\tau_{\left.b^{*}\right\}}^{-}\right.} \mathrm{e}^{-q t} \mathrm{E}_{Y_{t}}\left[\delta \int_{0}^{\sigma^{b^{*}}} \mathrm{e}^{-q s} \mathbf{1}_{\left\{U_{s}^{\left.b^{*} \in\left(b^{*}, \infty\right)\right\}}\right.} \mathrm{d} s-\frac{\delta}{q}\right] \\
& =\mathbf{1}_{\left\{t<\tau_{\left.b^{*}\right\}}^{-}\right.} \mathrm{e}^{-q t} \mathrm{E}_{Y_{t}}\left[\delta \int_{\kappa_{b^{*}}^{-}}^{\sigma^{b^{*}}} \mathrm{e}^{-q s} \mathbf{1}_{\left\{U_{s}^{b^{*}} \in\left(b^{*}, \infty\right)\right\}} \mathrm{d} s-\frac{\delta}{q} \mathrm{e}^{-q \kappa_{b^{*}}^{-}}\right] \\
& =\mathbf{1}_{\left\{t<\tau_{\left.b^{*}\right\}}^{-}\right.} \mathrm{e}^{-q t} \mathbb{E}_{Y_{t}}\left[\mathrm{e}^{-q \tau_{b^{*}}^{-}}\left(v_{b^{*}}\left(Y_{\tau_{b^{*}}^{-}}\right)-\frac{\delta}{q}\right)\right],
\end{aligned}
$$

where in the last line we used the fact that, given $Y_{0}=U_{0},\left\{Y_{t}, 0 \leq t \leq \tau_{b^{*}}^{-}\right\}$is equal in law to $\left\{U_{t}^{b^{*}}, 0 \leq t \leq \kappa_{b^{*}}^{-}\right\}$

We now continue with the proof of the lemma. It is easily seen that condition (14) is equivalent to

$$
\begin{gathered}
(\Gamma-q) v_{b^{*}}(x) \leq 0 \quad \text { if } v_{b^{*}}^{\prime}(x) \geq 1 \\
(\Gamma-q) v_{b^{*}}(x)-\delta v_{b^{*}}^{\prime}(x)+\delta \leq 0 \quad \text { if } v_{b^{*}}^{\prime}(x)<1 .
\end{gathered}
$$

Suppose now that (19) holds. If $v_{b^{*}}^{\prime}(x)>1$ then (19) implies that $x \leq b^{*}$ and so, by (20), $(\Gamma-q) v_{b^{*}}(x)=0$. If $v_{b^{*}}^{\prime}(x)<1$ then (19) implies that $x>b^{*}$ and so, by (20), $(\Gamma-q) v_{b^{*}}(x)-\delta v_{b^{*}}^{\prime}(x)+\delta=0$. If $v_{b^{*}}^{\prime}(x)=1$ then we have, by $(20),(\Gamma-q) v_{b^{*}}(x)=0$. Hence, (21) holds.

Suppose now that (21) holds. Let $0<x \leq b^{*}$, and suppose that $v_{b^{*}}^{\prime}(x)<1$. Then (20) and (21) imply that $-\delta v_{b^{*}}^{\prime}(x)+\delta \leq 0$, which implies that $v_{b^{*}}^{\prime}(x) \geq 1$, yielding a contradiction. Hence, we deduce that $v_{b^{*}}^{\prime}(x) \geq 1$. Now let $x>b^{*}$ and suppose that $v_{b^{*}}^{\prime}(x)>1$. Then (20) and (21) imply that $\delta v_{b^{*}}^{\prime}(x)-\delta \leq 0$, which implies that $v_{b^{*}}^{\prime}(x) \leq 1$, yielding a contradiction. Hence, we deduce that $v_{b^{*}}^{\prime}(x) \leq 1$. 
The following lemma forms the most difficult part of the proof of the main theorem. It is here that Lemma 2 and, thus, the assumption of complete monotonicity on the density of the Lévy measure is most crucially needed. In particular, we need here the representation of the scale function as the difference of an exponential term and a completely monotone function (cf. Lemma 2), instead of the weaker property that the derivative of the scale function is logconvex (see also the discussion below Theorem 1.2 of [28]) or the even weaker property that the derivative of the scale function is increasing on $\left(a^{*}, \infty\right)$ (see also Theorem 2 of [25] and Example 1 below); note that the latter is already sufficient for optimality of the barrier strategy for the control problem with general admissible strategies.

Lemma 7. Suppose that the Lévy measure has a completely monotone density. Then the function $v_{b^{*}}$ satisfies (19).

Proof. Suppose first that $b^{*}=0$. In other words, from Lemma 3, assume that either

(i) $\sigma>0$ and $\varphi(q) \geq 2 \delta / \sigma^{2}$, or

(ii) $\sigma=0, v(0, \infty)<\infty$, and $\varphi(q) \geq \delta(q+v(0, \infty)) / c(c-\delta)$.

Then, for $x>0$, we deduce from (15) that

$$
v_{b^{*}}^{\prime}(x)=v_{0}^{\prime}(x)=-\delta\left(\mathbb{W}^{(q)}(x)-\frac{1}{\varphi(q)} \mathbb{W}^{(q)^{\prime}}(x)\right) .
$$

By the decomposition of the scale function given in Lemma 2, $v_{0}^{\prime}$ is completely monotone and, thus, in particular decreasing on $(0, \infty)$. Hence, if $b^{*}=0$, it is enough to show that $v_{0}^{\prime}(0+) \leq 1$, or, equivalently,

$$
\frac{\delta \mathbb{W}^{(q)^{\prime}}(0+)}{1+\delta \mathbb{W}^{(q)}(0)} \leq \varphi(q)
$$

Taking account of Lemma 1 we see that this requirement is automatically satisfied in cases (i) and (ii). Hence, we have proved (19) if $b^{*}=0$.

Assume now that $b^{*}>0$. Then, for $x \leq b^{*}, v_{b^{*}}^{\prime}(x)=W^{(q) \prime}(x) / W^{(q)^{\prime}}\left(b^{*}\right)$. From this, it follows that $v_{b^{*}}^{\prime}(x) \leq 1$ since, by Lemma $3, b^{*} \leq a^{*}$ and, by Lemma $2, W^{(q)^{\prime}}$ is decreasing for $x \leq b^{*}$.

Suppose now that $x>b^{*}$. Differentiating twice the first displayed equation in Section 8 of [22] gives us the identity

$$
\delta \int_{0}^{x} \mathbb{W}^{(q)^{\prime}}(x-y) W^{(q) \prime}(y) \mathrm{d} y=\left(1-\delta W^{(q)}(0)\right) \mathbb{W}^{(q)^{\prime}}(x)-\left(1+\delta \mathbb{W}^{(q)}(0)\right) W^{(q) \prime}(x) .
$$

Hence, revisiting (16) we obtain the expression

$$
v_{b^{*}}^{\prime}(x)=-\delta \mathbb{W}^{(q)}\left(x-b^{*}\right)+\frac{\left(1-\delta W^{(q)}(0)\right) \mathbb{W}^{(q) \prime}(x)-\delta \int_{0}^{b^{*}} \mathbb{W}^{(q) \prime}(x-y) W^{(q) \prime}(y) \mathrm{d} y}{W^{(q)^{\prime}}\left(b^{*}\right)} .
$$

Appealing to Lemma 2 and writing $\mathbb{W}^{(q)}(x)=\varphi^{\prime}(q) \mathrm{e}^{\varphi(q) x}-f(x)$, where $f$ is completely 
monotone, we obtain

$$
\begin{array}{r}
v_{b^{*}}^{\prime}(x)=\delta f\left(x-b^{*}\right)+\frac{-\left(1-\delta W^{(q)}(0)\right) f^{\prime}(x)+\delta \int_{0}^{b^{*}} f^{\prime}(x-y) W^{(q) \prime}(y) \mathrm{d} y}{W^{(q) \prime}\left(b^{*}\right)} \\
-\delta \varphi^{\prime}(q) \mathrm{e}^{\varphi(q)\left(x-b^{*}\right)}+\frac{1}{W^{(q)^{\prime}\left(b^{*}\right)}}\left(\left(1-\delta W^{(q)}(0)\right) \varphi^{\prime}(q) \varphi(q) \mathrm{e}^{\varphi(q) x}\right. \\
\left.-\delta \int_{0}^{b^{*}} \varphi^{\prime}(q) \varphi(q) \mathrm{e}^{\varphi(q)(x-y)} W^{(q) \prime}(y) \mathrm{d} y\right) .
\end{array}
$$

Using (13) and recalling that $\varphi(q)>\Phi(q)$, we also have

$$
\begin{aligned}
\int_{0}^{b^{*}} \mathrm{e}^{-\varphi(q) y} W^{(q) \prime}(y) \mathrm{d} y & =-\int_{b^{*}}^{\infty} \mathrm{e}^{-\varphi(q) y} W^{(q) \prime}(y) \mathrm{d} y+\int_{0}^{\infty} \mathrm{e}^{-\varphi(q) y} W^{(q) \prime}(y) \mathrm{d} y \\
& =-\int_{b^{*}}^{\infty} \mathrm{e}^{-\varphi(q) y} W^{(q) \prime}(y) \mathrm{d} y+\frac{1}{\delta}-W^{(q)}(0) .
\end{aligned}
$$

Hence, this gives

$$
\begin{aligned}
v_{b^{*}}^{\prime}(x)= & \delta f\left(x-b^{*}\right)+\frac{-\left(1-\delta W^{(q)}(0)\right) f^{\prime}(x)+\delta \int_{0}^{b^{*}} f^{\prime}(x-y) W^{(q) \prime}(y) \mathrm{d} y}{W^{(q)^{\prime}}\left(b^{*}\right)} \\
& -\delta \varphi^{\prime}(q) \mathrm{e}^{\varphi(q)\left(x-b^{*}\right)}+\frac{\delta \mathrm{e}^{\varphi(q) x} \varphi^{\prime}(q) \varphi(q) \int_{b^{*}}^{\infty} \mathrm{e}^{-\varphi(q) y} W^{(q)^{\prime}}(y) \mathrm{d} y}{W^{(q) \prime}\left(b^{*}\right)}
\end{aligned}
$$

and recalling that

$$
W^{(q) \prime}\left(b^{*}\right)=h\left(b^{*}\right)=\varphi(q) \mathrm{e}^{\varphi(q) b^{*}} \int_{b^{*}}^{\infty} \mathrm{e}^{-\varphi(q) y} W^{(q) \prime}(y) \mathrm{d} y,
$$

we obtain the simpler expression

$$
v_{b^{*}}^{\prime}(x)=\delta f\left(x-b^{*}\right)+\frac{-\left(1-\delta W^{(q)}(0)\right) f^{\prime}(x)+\delta \int_{0}^{b^{*}} f^{\prime}(x-y) W^{(q) \prime}(y) \mathrm{d} y}{W^{(q)^{\prime}}\left(b^{*}\right)} .
$$

Since $f$ is completely monotone, by Bernstein's theorem (cf. [13, Theorem 1a, Section XIII.4]), it can be written in the form $f(x)=\int_{0}^{\infty} \mathrm{e}^{-x t} \mu(\mathrm{d} t)$ for some measure $\mu$. Therefore, using Tonelli's theorem (cf. [32, Theorem 4.1.5]), we arrive at the identity

$$
v_{b^{*}}^{\prime}(x)=\int_{0}^{\infty} \mathrm{e}^{-x t}\left\{\delta \mathrm{e}^{b^{*} t}+\frac{1-\delta W^{(q)}(0)}{W^{(q)^{\prime}}\left(b^{*}\right)} t-\frac{\delta}{W^{(q)^{\prime}}\left(b^{*}\right)} \int_{0}^{b^{*}} t \mathrm{e}^{y t} W^{(q)^{\prime}}(y) \mathrm{d} y\right\} \mu(\mathrm{d} t) .
$$

For $t>0$, denote by $g(t)$ the expression in the curly brackets. Thus,

$$
g^{\prime \prime}(t)=\delta\left(b^{*}\right)^{2} \mathrm{e}^{b^{*} t}-\frac{2 \delta}{W^{(q)^{\prime}\left(b^{*}\right)}} \int_{0}^{b^{*}} y \mathrm{e}^{y t} W^{(q)^{\prime}}(y) \mathrm{d} y-\frac{\delta t}{W^{(q)^{\prime}}\left(b^{*}\right)} \int_{0}^{b^{*}} y^{2} \mathrm{e}^{y t} W^{(q)^{\prime}}(y) \mathrm{d} y .
$$

Since $W^{(q)^{\prime}}(y) \geq W^{(q)^{\prime}}\left(b^{*}\right)$ for $y \in\left(0, b^{*}\right)$, we have, also using integration by parts,

$$
\frac{\delta t}{W^{(q)^{\prime}}\left(b^{*}\right)} \int_{0}^{b^{*}} y^{2} \mathrm{e}^{y t} W^{(q) \prime}(y) \mathrm{d} y \geq \delta t \int_{0}^{b^{*}} y^{2} \mathrm{e}^{y t} \mathrm{~d} y=\left.\delta y^{2} \mathrm{e}^{y t}\right|_{0} ^{b^{*}}-2 \delta \int_{0}^{b^{*}} y \mathrm{e}^{y t} \mathrm{~d} y,
$$


and, hence, again using the fact that $W^{(q)^{\prime}}(y) \geq W^{(q) \prime}\left(b^{*}\right)$ for $y \in\left(0, b^{*}\right)$,

$$
g^{\prime \prime}(t) \leq-\frac{2 \delta}{W^{(q) \prime}\left(b^{*}\right)} \int_{0}^{b^{*}} y \mathrm{e}^{y t} W^{(q) \prime}(y) \mathrm{d} y+2 \delta \int_{0}^{b^{*}} y \mathrm{e}^{y t} \mathrm{~d} y \leq 0 .
$$

In conclusion, $g$ is a concave function, and in particular there exists $0 \leq p_{1} \leq \infty$ such that $g$ is increasing on $\left(0, p_{1}\right)$ and decreasing on $\left(p_{1}, \infty\right)$. Since $g(0)=\delta$, it follows that there exists $0<p_{2} \leq \infty$ such that $g$ is positive on $\left(0, p_{2}\right)$ and negative on $\left(p_{2}, \infty\right)$. Consequently, $\mathrm{e}^{-\left(x-b^{*}\right) t} g(t) \geq \mathrm{e}^{-\left(x-b^{*}\right) p_{2}} g(t)$ for all $t>0$ and, thus (noting that we are allowed to switch the derivative and the integral),

$$
\begin{aligned}
v_{b^{*}}^{\prime \prime}(x) & =-\int_{0}^{\infty} \mathrm{e}^{-\left(x-b^{*}\right) t} \mathrm{e}^{-b^{*} t} \operatorname{tg}(t) \mathrm{d} t \\
& \leq-\mathrm{e}^{-\left(x-b^{*}\right) p_{2}} \int_{0}^{\infty} \mathrm{e}^{-b^{*} t} \operatorname{tg}(t) \mathrm{d} t \\
& =\mathrm{e}^{-\left(x-b^{*}\right) p_{2}} v_{b^{*}}^{\prime \prime}\left(b^{*}+\right) .
\end{aligned}
$$

From (18) we easily deduce that

$$
v_{b^{*}}^{\prime \prime}\left(b^{*}+\right)=\frac{\left(1+\delta \mathbb{W}^{(q)}(0)\right) W^{(q) \prime \prime}\left(b^{*}\right)}{W^{(q) \prime}\left(b^{*}\right)} \leq 0,
$$

where the inequality is a result of the fact that $b^{*}<a^{*}$ and, hence, by Lemma $2, W^{(q) \prime \prime}\left(b^{*}\right) \leq 0$. In combination with (23), it follows that $v_{b^{*}}^{\prime}$ is decreasing on $\left(b^{*}, \infty\right)$ and since $v_{b^{*}}^{\prime}\left(b^{*}\right)=1$, we deduce that $v_{b^{*}}^{\prime}(x) \leq 1$ for $x>b^{*}$, as required.

Finally, we can put all the pieces together to establish our main result.

Proof of Theorem 1. By Lemmas 6 and 7, it follows that $v_{b^{*}}$ satisfies (14). Since, in addition, $v_{b^{*}}$ is right continuous at 0 and is sufficiently smooth by Lemma 5 , it follows by Lemma 4 that $v_{b^{*}}$ equals the optimal value function $v_{*}$ and, thus, the refraction strategy with threshold level $b^{*}$ is an optimal strategy.

Example 1. Here we give the example mentioned at the end of Section 1. Let $v(\mathrm{~d} z)=$ $10 z \mathrm{e}^{-z} \mathrm{~d} z, \gamma=20.67-\int_{0}^{1} z v(\mathrm{~d} z)$, and $q=0.1$. That is, $X$ is a Cramér-Lundberg risk process with premium rate 20.67, jump arrival rate 10 , and a $\operatorname{Gamma}(2,1)$ claim distribution. The scale function for $X$, via the method of partial fractions, is given by

$$
W^{(q)}(x)=\sum_{i=1}^{3} D_{i} \mathrm{e}^{\theta_{i} x}, \quad x \geq 0,
$$

where $\left\{\theta_{i}: i=1,2,3\right\}$ are the (distinct) roots of $\lambda \mapsto \psi(\theta)-q$ with $\theta_{1}>0$ and $\theta_{2}, \theta_{3}<$ 0 , and $\left\{D_{i}: i=1,2,3\right\}$ are given by $D_{i}=1 / \psi^{\prime}\left(\theta_{i}\right)$. The scale function of $Y$ can be similarly calculated. If one plots the second derivative of $W^{(q)}$ then one sees that $W^{(q) \prime \prime}(x)>0$ on $(0, \infty)$ and, therefore, by Theorem 2 of [25], the reflection strategy at the barrier 0 is optimal for the control problem with general admissible dividend strategies. If we now consider the case with absolutely continuous dividend strategies and choose the upper bound $\delta$ equal to 20.59 , then we can check that the function $h$ is strictly increasing and, thus, $v_{0}(0)=W^{(q)}(0) / h(0)>W^{(q)}(0) / h(b)=v_{b}(0)$ for all $b>0$. This means that the only refraction strategy that can be optimal is the one with threshold level equal to 0 . However, one can calculate that $v_{0}^{\prime}(3.15)=1.0005>1$ and so $v_{0}$ does not satisfy (19) and, consequently, by Lemma $6, v_{0}$ does not satisfy (14). Since we are in the Cramér-Lundberg setting with a continuous claim distribution, we can deduce from Theorem 2.32 of [31] that the optimal value 
function $v_{*}$ has to satisfy (14) and, therefore, $v_{0} \neq v_{*}$. We conclude that for this particular example we have the remarkable property that the optimal strategy in the case with no extra restrictions on the controls is to always pay out the maximum amount of dividends that is allowed, whereas in the restricted case with this particular value of $\delta$, it is not optimal to always pay out dividends at the maximum rate. Furthermore, as for this example $W^{(q) \prime}$ is increasing on $\left(a^{*}, \infty\right)$, it follows that this latter condition is not sufficient for a refraction strategy to be optimal amongst the class of dividend strategies satisfying (3) and (4); this is in contrast with the singular optimal dividends problem; cf. Theorem 2 of [25].

On a final note, we present the following corollary of the proof of Theorem 1, giving a characterisation of the optimal threshold; cf. Lemma 3.

Corollary 1. The threshold for the optimal refraction strategy in Theorem 1 is characterised as the unique point $b^{*} \in\left[0, a^{*}\right)$ such that

$$
b^{*}=\sup \{b \geq 0: h(b) \leq h(x) \text { for all } x \geq 0\}
$$

where

$$
h(b)=\varphi(q) \mathrm{e}^{\varphi(q) b} \int_{b}^{\infty} \mathrm{e}^{-\varphi(q) y} W^{(q) \prime}(y) \mathrm{d} y .
$$

Moreover, $b^{*}>0$ if and only if

(i) $\sigma>0$ and $\varphi(q)<2 \delta / \sigma^{2}$,

(ii) $\sigma=0, v(0, \infty)<\infty$, and $\varphi(q)<\delta(v(0, \infty)+q) / c(c-\delta)$, or

(iii) $\sigma=0$ and $v(0, \infty)=\infty$;

otherwise, $b^{*}=0$.

\section{Appendix A. Proof of Lemma 4}

By the definition of $v_{*}$, it follows that $v_{\hat{\pi}}(x) \leq v_{*}(x)$ for all $x \geq 0$. We write $w:=v_{\hat{\pi}}$ and show that $w(x) \geq v_{\pi}(x)$ for all $\pi \in \Pi$ and all $x \geq 0$. First we suppose that $x>0$. We define, for $\pi \in \Pi$, the stopping time $\sigma_{0}^{\pi}$ by $\sigma_{0}^{\pi}=\inf \left\{t>0: U_{t}^{\pi} \leq 0\right\}$ and denote by $\Pi_{0}$ the following set of admissible dividend strategies:

$$
\Pi_{0}=\left\{\pi \in \Pi: v_{\pi}(x)=\mathrm{E}_{x}\left[\int_{0}^{\sigma_{0}^{\pi}} \mathrm{e}^{-q t} \mathrm{~d} L_{t}^{\pi}\right] \text { for all } x>0\right\} .
$$

We claim that any $\pi \in \Pi$ can be approximated by dividend strategies from $\Pi_{0}$ in the sense that, for all $\varepsilon>0$, there exists $\pi_{\varepsilon} \in \Pi_{0}$ such that $v_{\pi}(x) \leq v_{\pi_{\varepsilon}}(x)+\varepsilon$ and, therefore, it is enough to show that $w(x) \geq v_{\pi}(x)$ for all $\pi \in \Pi_{0}$. Indeed, we can take $\pi_{\varepsilon}$ to be the strategy where you do not pay out any dividends until the stopping time $\kappa:=\inf \left\{t>0: L_{t}^{\pi} \geq \varepsilon\right\}$, then from that time point $\kappa$ onwards follow the same strategy as $\pi$ until ruin occurs for the latter strategy, at which point you stop paying out dividends. Note that $\pi_{\varepsilon} \in \Pi_{0}$ because if $\sigma_{0}^{\pi_{\varepsilon}}<\kappa$ then $\sigma_{0}^{\pi_{\varepsilon}}=\sigma^{\pi_{\varepsilon}}$ since, until the first dividend payment is made, the process $U^{\pi_{\varepsilon}}$ is equal to $X$ and, for the spectrally negative Lévy process $X$, the first entry time in $(-\infty, 0]$ is equal almost surely to the first entry time in $(-\infty, 0)$, provided that $X_{0}>0$. Furthermore, if $\sigma_{0}^{\pi_{\varepsilon}} \geq \kappa$ and $\kappa<\infty$, then $\sigma_{0}^{\pi_{\varepsilon}} \geq \sigma^{\pi}$ and so, by construction, there are no dividends paid out in the time interval $\left(\sigma_{0}^{\pi_{\varepsilon}}, \sigma^{\pi_{\varepsilon}}\right)$. 
We now assume without loss of generality that $\pi \in \Pi_{0}$. Let $\left(T_{n}\right)_{n \in \mathbb{N}}$ be the sequence of stopping times defined by $T_{n}=\inf \left\{t>0: U_{t}^{\pi}>n\right.$ or $\left.U_{t}^{\pi}<1 / n\right\}$. Since $U^{\pi}$ is a semimartingale and $w$ is sufficiently smooth, we can use the change of variables/Itô's formula (cf. [29, Theorems II.31 and II.32]) on $\mathrm{e}^{-q\left(t \wedge T_{n}\right)} w\left(U_{t \wedge T_{n}}^{\pi}\right)$ to deduce, under $\mathrm{P}_{x}$,

$$
\begin{aligned}
\mathrm{e}^{-q\left(t \wedge T_{n}\right)} w\left(U_{t \wedge T_{n}}^{\pi}\right)-w(x)= & -\int_{0+}^{t \wedge T_{n}} \mathrm{e}^{-q s} q w\left(U_{s-}^{\pi}\right) \mathrm{d} s+\int_{0+}^{t \wedge T_{n}} \mathrm{e}^{-q s} w^{\prime}\left(U_{s-}^{\pi}\right) \mathrm{d}\left(X_{s}-L_{s}^{\pi}\right) \\
& +\sum_{0<s \leq t \wedge T_{n}} \mathrm{e}^{-q s}\left[\Delta w\left(U_{s-}^{\pi}+\Delta X_{S}\right)-w^{\prime}\left(U_{s-}^{\pi}\right) \Delta X_{s}\right],
\end{aligned}
$$

where we have used the notation $\Delta U_{s}^{\pi}=U_{s}^{\pi}-U_{s-}^{\pi}$ and $\Delta w\left(U_{s}^{\pi}\right)=w\left(U_{s}^{\pi}\right)-w\left(U_{s-}^{\pi}\right)$. Rewriting the above equation leads to

$$
\begin{aligned}
\mathrm{e}^{-q\left(t \wedge T_{n}\right)} w\left(U_{t \wedge T_{n}}^{\pi}\right)-w(x) & \int_{0+}^{t \wedge T_{n}} \mathrm{e}^{-q s}(\Gamma-q) w\left(U_{s-}^{\pi}\right) \mathrm{d} s-\int_{0+}^{t \wedge T_{n}} \mathrm{e}^{-q s} w^{\prime}\left(U_{s-}^{\pi}\right) \mathrm{d} L_{s}^{\pi} \\
+ & \left\{\int_{0+}^{t \wedge T_{n}} \mathrm{e}^{-q s} w^{\prime}\left(U_{s-}^{\pi}\right) \mathrm{d}\left[X_{s}-\left(c-\int_{0}^{1} x v(\mathrm{~d} x)\right) s-\sum_{0<u \leq s} \Delta X_{u} \mathbf{1}_{\left\{\left|\Delta X_{u}\right|>1\right\}}\right]\right\} \\
+ & \left\{\sum_{0<s \leq t \wedge T_{n}} \mathrm{e}^{-q s}\left[\Delta w\left(U_{s-}^{\pi}+\Delta X_{s}\right)-w^{\prime}\left(U_{s-}^{\pi}\right) \Delta X_{s} \mathbf{1}_{\left.\left\{\left|\Delta X_{s}\right| \leq 1\right\}\right]}\right]\right. \\
& \left.\quad-\int_{0+}^{t \wedge T_{n}} \int_{0+}^{\infty} \mathrm{e}^{-q s}\left[w\left(U_{s-}^{\pi}-y\right)-w\left(U_{s-}^{\pi}\right)+w^{\prime}\left(U_{s-}^{\pi}\right) y \mathbf{1}_{\{0<y \leq 1\}}\right] v(\mathrm{~d} y) \mathrm{d} s\right\} .
\end{aligned}
$$

By the Lévy-Itô decomposition, the expression between the first pair of curly brackets is a zeromean martingale and, by the compensation formula (cf. [21, Corollary 4.6]), the expression between the second pair of curly brackets is also a zero-mean martingale. Hence, we derive at

$$
\begin{aligned}
w(x)= & -\int_{0}^{t \wedge T_{n}} \mathrm{e}^{-q s}\left[(\Gamma-q) w\left(U_{s-}^{\pi}\right)-\ell^{\pi}(s) w^{\prime}\left(U_{s-}^{\pi}\right)+\ell^{\pi}(s)\right] \mathrm{d} s \\
& +\int_{0}^{t \wedge T_{n}} \mathrm{e}^{-q s} \ell^{\pi}(s) \mathrm{d} s+\mathrm{e}^{-q\left(t \wedge T_{n}\right)} w\left(U_{t \wedge T_{n}}^{\pi}\right)+M_{t},
\end{aligned}
$$

where $\left\{M_{t}: t \geq 0\right\}$ is a zero-mean $\mathrm{P}_{x}$-martingale. Using $w \geq 0$ and (14) leads to (since $\left.0 \leq \ell_{s}^{\pi} \leq \delta\right)$

$$
w(x) \geq \int_{0}^{t \wedge T_{n}} \mathrm{e}^{-q s} \ell^{\pi}(s) \mathrm{d} s+M_{t} .
$$

Now taking expectations and letting $t$ and $n$ go to $\infty$ and using the monotone convergence theorem we obtain, noting that $T_{n} \nearrow \sigma_{0}^{\pi}, \mathrm{P}_{x}$-almost surely and that $\pi \in \Pi_{0}$,

$$
w(x) \geq \mathrm{E}_{x}\left[\int_{0}^{\sigma^{\pi}} \mathrm{e}^{-q s} \ell^{\pi}(s) \mathrm{d} s\right]=v_{\pi}(x) .
$$

Hence, we have proved $w(x) \geq v_{*}(x)$ for all $x>0$. 
To finish the proof, note that $v_{*}$ is an increasing function (in the weak sense) and, hence, because $w$ is right continuous at zero, $v_{*}(0) \leq \lim _{x \downarrow 0} v_{*}(x) \leq \lim _{x \downarrow 0} w(x)=w(0)$.

\section{References}

[1] Albrecher, H. and Thonhauser, S. (2009). Optimality results for dividend problems in insurance. Rev. $R$. Acad. Cienc. Exactas Fís. Nat. Ser. A Math. RACSAM 103, 295-320.

[2] Asmussen, S. And Taksar, M. (1997). Controlled diffusion models for optimal dividend pay-out. Insurance Math. Econom. 20, 1-15.

[3] AvanZI, B. (2009). Strategies for dividend distribution: a review. N. Amer. Actuarial J. 13, 217-251.

[4] Avram, F., Palmowski, Z. and Pistorius, M. R. (2007). On the optimal dividend problem for a spectrally negative Lévy process. Ann. Appl. Prob. 17, 156-180.

[5] Azcue, P. and Muler, N. (2005). Optimal reinsurance and dividend distribution policies in the CramérLundberg model. Math. Finance 15, 261-308.

[6] Bayraktar, E. and Egami, M. (2008). Optimizing venture capital investments in a jump diffusion model. Math. Methods Operat. Res. 67, 21-42.

[7] BelhaJ, M. (2010). Optimal dividend payments when cash reserves follow a jump-diffusion process. Math. Finance 20, 313-325.

[8] Biffis, E. And Kyprianou, A. E. (2010). A note on scale functions and the time value of ruin for Lévy insurance risk processes. Insurance Math. Econom. 46, 85-91.

[9] Boguslavaskaya, E. (2006). Optimization problems in financial mathematics: explicit solutions for diffusion models. Doctoral Thesis, University of Amsterdam.

[10] Chan, T., Kyprianou, A. E. And Savov, M. (2011). Smoothness of scale functions for spectrally negative Lévy processes. Prob. Theory Relat. Fields 150, 691-708.

[11] De Finetri, B. (1957). Su un'impostazione alternativa della teoria collettiva del rischio. In Transactions of the 15th International Congress of Actuaries, Vol. II, pp. 433-443.

[12] Doney, R. A. And Kyprianou, A. E. (2006). Overshoots and undershoots of Lévy processes. Ann. Appl. Prob. 16, 91-106.

[13] Feller, W. (1971). An Introduction to Probability Theory and Its Applications, Vol. II, 2nd edn. John Wiley, New York.

[14] Gerber, H. U. (1969). Entscheidungskriterien für den zusammengesetzten Poisson-prozess. Mit. Verein. Schweiz. Versicherungsmath. 69, 185-227.

[15] Gerber, H. (1974). The dilemma between dividends and safety and a generalization of the Lundberg-Cramér formulas. Scand. Actuarial J. 1974, 46-57.

[16] Gerber, H. U. and Shiu, E. S. W. (2006). On optimal dividend strategies in the compound Poisson model. N. Amer. Actuarial J. 10, 76-93.

[17] Huzak, M., Perman, M., Šıkić, H. and Vondraček, Z. (2004). Ruin probabilities for competing claim processes. J. Appl. Prob. 41, 679-690.

[18] Huzak, M., Perman, M., ŠIKić, H. and VondračEK, Z. (2004). Ruin probabilities and decompositions for general perturbed risk processes. Ann. Appl. Prob. 14, 1378-1397.

[19] Jeanblanc-Picqué, M. And Shiryaev, A. N. (1995). Optimization of the flow of dividends. Russian Math. Surveys 50, 257-277.

[20] Klüppelberg, C., Kyprianou A. E. And Maller, R. A. (2004). Ruin probabilities and overshoots for general Lévy insurance risk processes. Ann. Appl. Prob. 14, 1766-1801.

[21] Kyprianou, A. E. (2006). Introductory Lectures on Fluctuations of Lévy Processes with Applications. Springer, Berlin.

[22] Kyprianou, A. E. And Loeffen, R. L. (2010). Refracted Lévy processes. Ann. Inst. H. Poincaré Prob. Statist. 46, 24-44.

[23] Kyprianou, A. E. and Palmowski, Z. (2007). Distributional study of de Finetti's dividend problem for a general Lévy insurance risk process. J. Appl. Prob. 44, 349-365.

[24] Kyprianou, A. E., Rivero, V. And Song, R. (2010). Convexity and smoothness of scale functions and de Finetti's control problem. J. Theoret. Prob. 23, 547-564.

[25] Loeffen, R. L. (2008). On optimality of the barrier strategy in de Finetti's dividend problem for spectrally negative Lévy processes. Ann. Appl. Prob. 18, 1669-1680.

[26] LoefFen, R. L. (2009). An optimal dividends problem with a terminal value for spectrally negative Lévy processes with a completely monotone jump density. J. Appl. Prob. 46, 85-98.

[27] Loeffen, R. L. (2009). An optimal dividends problem with transaction costs for spectrally negative Lévy processes. Insurance Math. Econom. 45, 41-48.

[28] Loeffen, R. L. And Renaud, J.-F. (2010). De Finetti's optimal dividends problem with an affine penalty function at ruin. Insurance Math. Econom. 46, 98-108. 
[29] Protter, P. E. (2005). Stochastic Integration and Differential Equations, 2nd edn. Springer, Berlin.

[30] RADNER, R. AND SHEPP, L. (1996). Risk vs. profit potential: a model for corporate strategy. J. Econom. Dynamics Control 20, 1373-1393.

[31] Schmidui, H. (2008). Stochastic Control in Insurance. Springer, London.

[32] Stroock, D. W. (1999). A Concise Introduction to the Theory of Integration, 3rd edn. Birkhäuser, Boston, MA.

[33] Williams, D. (1991). Probability with Martingales. Cambridge University Press. 